\title{
Single-Walled Carbon Nanotube Network Field Effect Transistor as a Humidity Sensor
}

\author{
Prasantha R. Mudimela, ${ }^{1,2}$ Kestutis Grigoras, ${ }^{3}$ Ilya V. Anoshkin, ${ }^{1}$ \\ Aapo Varpula, ${ }^{3}$ Vladimir Ermolov, ${ }^{4,5}$ Anton S. Anisimov, ${ }^{1,6}$ Albert G. Nasibulin, ${ }^{1}$ \\ Sergey Novikov, ${ }^{7}$ and Esko I. Kauppinen ${ }^{1}$ \\ ${ }^{1}$ NanoMaterials Group, Department of Applied Physics, Aalto University, P.O. Box 15100, 00076 Aalto, Finland \\ ${ }^{2}$ IACQER Center for Research and Development on Nanotechnology, Malla Reddy Engineering College, Secunderabad 500014, India \\ ${ }^{3}$ Microsystems and Nanoelectronics, VTT Technical Research Centre of Finland, P.O. Box 1000, 02044 VTT, Finland \\ ${ }^{4}$ Nokia Research Centre, Helsinki, Finland \\ ${ }^{5}$ VTT Technical research centre of Finland, Tietotie 3, 02044 Espoo, Finland \\ ${ }^{6}$ Canatu Oy, Konalankuja 5, 00390 Helsinki, Finland \\ ${ }^{7}$ Department of Materials Science and Technology, Aalto University, P.O. Box 13500, 00076 Aalto, Finland
}

Correspondence should be addressed to Kestutis Grigoras, kestas.grigoras@gmail.com and Albert G. Nasibulin, albert.nasibulin@aalto.fi

Received 10 January 2012; Accepted 19 March 2012

Academic Editor: Andrea Cusano

Copyright (c) 2012 Prasantha R. Mudimela et al. This is an open access article distributed under the Creative Commons Attribution License, which permits unrestricted use, distribution, and reproduction in any medium, provided the original work is properly cited.

Single-walled carbon nanotube network field effect transistors were fabricated and studied as humidity sensors. Sensing responses were altered by changing the gate voltage. At the open channel state (negative gate voltage), humidity pulse resulted in the decrease of the source-drain current, and, vice versa, the increase in the source-drain current was observed at the positive gate voltage. This effect was explained by the electron-donating nature of water molecules. The operation speed and signal intensity was found to be dependent on the gate voltage polarity. The positive or negative change in current with humidity pulse at zero-gate voltage was found to depend on the previous state of the gate electrode (positive or negative voltage, respectively). Those characteristics were explained by the charge traps in the gate dielectric altering the effective gate voltage, which influenced the operation of field effect transistor.

\section{Introduction}

Single-walled carbon nanotubes (SWCNTs) have a great promise for many applications due to their unique electronic and optical properties [1]. Depending on their chiral structure, the properties of the SWCNTs can vary from semiconducting to metallic [2]. At the moment, controlled synthesis of SWCNTs with uniform chirality is still a challenge. As a result, the reproducibility of the single tube devices due to their chirality deviations has not been achieved. Also, the alignment and positioning of SWCNTs are very complicated and require time and resource-consuming electron beam lithography. Nevertheless, in random SWCNT networks, the properties averaged over a large number of tubes suppress the effects of the SWCNT chirality variation and allows reaching reproducible results [3]. These networks can be easily prepared by various methods: by a direct growth on a catalyzed substrate [4], by depositions onto substrates either from a solution of the suspended SWCNTs [5], or from the gas phase synthesised by an aerosol method $[6,7]$. The SWCNT networks are of low cost and exhibit good transparency and high conductivity, making them ideal candidates for various potential applications such as thin film transistors [8,9], solar cells [10], displays [11], transparent conducting coatings $[12,13]$, and sensors [4]. The latter is very promising, however, little explored application of the SWCNT films, especially for humidity sensing.

Humidity sensors based on CNTs can be classified according to the change in the network resistivity, capacitance, or current in a field effect transistor (FET) configuration [14]. Most of the CNT humidity sensors are based on the 
resistivity change $[14,15]$, which can be explained by relative simplicity in the sensor fabrication and measurements. The humidity sensors were mainly prepared from the oxidised multiwalled carbon nanotubes (MWCNTs). It was observed that after the MWCNT treatment in a $\mathrm{HNO}_{3}-\mathrm{H}_{2} \mathrm{SO}_{4}$ mixture and ultrasonication the sensitivity of the sensors increased by 4 times [15]. Arrayed MWCNTs utilized for relative humidity $(\mathrm{RH})$ sensing in the range from 11 to $85 \%$ showed a resistance change of $362 \%$ with the response and recovery times of 64 and $51 \mathrm{~min}$, respectively [16]. The oxidized MWCNTs were also tested in alternating current (AC) devices in the frequency range from $500 \mathrm{~Hz}$ to $100 \mathrm{kHz}$. The sensor stability behavior was found to be 10 times better at $100 \mathrm{KHz}$ [17]. Vertical MWCNT arrays showed 6 times increase in the capacitance for the same humidity range at $50 \mathrm{kHz}$ [18].

Only a few papers have investigated humidity sensors based on CNT network FETs, which is apparently explained by the difficulties associated with the sensor fabrication, processing, and measurements. Individual CNTs covered by $\mathrm{Na}^{+}, \mathrm{K}^{+}$, and $\mathrm{Ca}^{2+}$ ion-exchanged Nafion polymer as sensitive and barrier material were used for water vapor detection [19]. Significant changes in source-drain current ( $\sim 10$ times) in the $\mathrm{RH}$ range from 13 to $93 \%$ were observed, but a complete understanding of the back-gated FET was missing. Liu et al. [20] utilized carboxylated MWCNTs aligned by AC pulses between FET electrodes. It was found that the interaction between water vapor and MWCNT networks is mainly determined by a weak physisorption. Several studies showed that water molecules acted as electron donors to the carbon nanotubes $[21,22]$.

In this work, we fabricated FETs based on random networks of SWCNTs synthesised by an aerosol (floating catalyst) chemical vapour deposition (CVD) method based on $\mathrm{CO}$ disproportionation at $1000^{\circ} \mathrm{C}$ on iron particles produced by ferrocene vapour decomposition $[13,23]$. The SWCNTs were directly deposited on to the FET substrate after the reaction and utilized without any additional treatment. In this work we show the effect of water vapour on the properties of pristine SWCNT in a FET circuit. The effect of humidity on the source-drain current of FET and its dependence on the gate voltage was investigated. It was found that the FET operation and sensitivity was determined by the charge traps due to water adsorption in the SWCNT film layer and gate insulator oxide.

\section{Experimental Methods}

The SWCNT random network FETs were fabricated on a heavily doped p-type $\mathrm{Si}$ substrate with $100 \mathrm{~nm} \mathrm{SiO}_{2}$ layer on top acting as a back-gate insulator. The whole Si wafer acted as a back-gated electrode, and $100 \mathrm{~nm}$ Al layer was sputtered on the bottom side to improve the contact to the gate electrode. Our samples were $1 \times 1 \mathrm{~cm}^{2}$ size chips obtained after dicing a 4-inch silicon wafer. The schematic view of transistor fabrication process is shown in Figure 1. First, SWCNT deposition areas were prepared by lithographically patterning AZ-5214 photoresist using a mask aligner (Karl Suss MA6/BA6) as shown in Figures 1(a), 1(b), and 1(c). The aerosol-synthesized SWCNTs were deposited at the outlet of the CVD reactor directly from the gas phase onto the chip by using an electrostatic precipitator described elsewhere [24]. SWCNT channel was obtained by lift-off technique: removing photoresist in acetone with subsequent rinsing in iso-propanol and water (Figure $1(\mathrm{~d})$ ). The process of the chip's fabrication did not include ultrasonication or any other treatments that could lead to the structural defects in the SWCNTs. Second lithography determined the source and drain electrode areas (Figure 1(e)). Electrical contacts were made from $\mathrm{Cr} / \mathrm{Au}(5 / 50 \mathrm{~nm}$ thick layers, resp.) layers deposited by electron beam evaporation (Figure $1(\mathrm{f})$ ) and patterned by liftoff (Figure 1(g)). FETs with different channel sizes (length $\times$ width) were fabricated on the same chip: from $5 \times 5 \mu \mathrm{m}^{2}$ to $50 \times 50 \mu \mathrm{m}^{2}$. The fabricated transistor chip was connected to the Au-contact pads by bonding wires. To determine the reproducibility of the fabricated FET, we measured the ON/OFF ratio and charge carrier mobilities for 10 identical devices with the channel size $50 \times 50 \mu \mathrm{m}^{2}$. It was found that the mobility varied from 5.5 to $20 \mathrm{~cm}^{2} \mathrm{~V}^{-1} \mathrm{~s}^{-1}$ while the ON/OFF ratio changed from $10^{1}$ to $10^{2}$ [25].

The humidity responses of the samples were measured in a gas control system shown in Figure 2. The clean dry compressed air provided by clean room facilities was used as a carrier gas. In the gas system, the air was split into two separate lines each controlled with a Celerity IN3XP mass flow controller (MFC). The MFCs were operated by a LabVIEW program. A gas-washing bottle with deionized water was used as a humidifier in one of the gas lines. After the humidifier, the gas lines were combined. The humidity and the temperature of the resulting gas mixture were measured with a Vaisala HUMITTER 50Y humidity probe. In the experiments the temperature of the air mixture was $20-23^{\circ} \mathrm{C}$. The produced gas mixture was connected to the test chamber at a gas flow rate of $0.4 \mathrm{~L} / \mathrm{min}$. Relative humidity $(\mathrm{RH})$ of $78 \%$ with a 67 min exposure time was used in most of the experiments unless otherwise mentioned.

Source-drain current $\left(I_{\mathrm{sd}}\right)$ was measured by sweeping the gate voltage from -10 to $10 \mathrm{~V}$ and back. A constant voltage of $600 \mathrm{mV}$ was applied between source and drain electrodes. For the electrical measurements, an HP 4155A semiconductor parameter analyser was used.

\section{Experimental Results and Discussion}

Usually, the synthesis of SWCNTs results in a mixture of metallic and semiconducting tubes. For the transistor's channel of SWCNT network, with a density below the metallic nanotube percolation level, the current is determined by the semiconducting nanotubes. The metallic SWCNT conductivity is almost independent of the gate voltage, while that of the semiconducting SWCNTs strongly depends on the gate voltage and usually exhibits a p-type channel behaviour of the transistor (Figure 3) [26]. Hence, at negative gate voltages, holes are accumulated in the CNTs, and the depletion barrier is decreased, reducing the source drain resistance and thus increasing the source-drain current $\left(I_{\mathrm{sd}}\right)$ through the channel. At positive gate voltages, due to the depletion of holes the channel resistance increases, and, therefore, $I_{s d}$ decreases. Figure 3 shows the results of the measurement of 


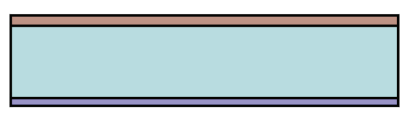

(a)

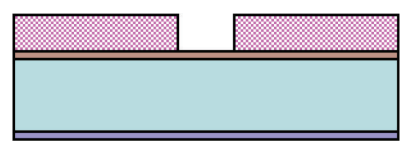

(b)

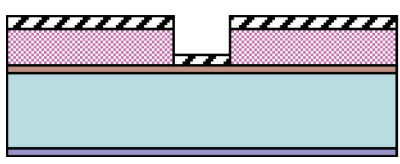

(c)



(d)

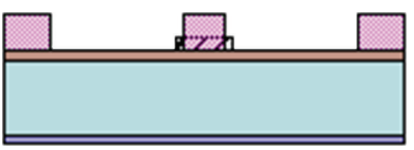

(e)

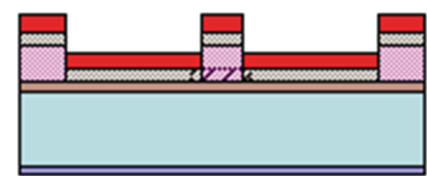

(f)

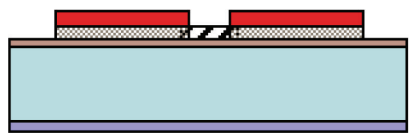

(g)

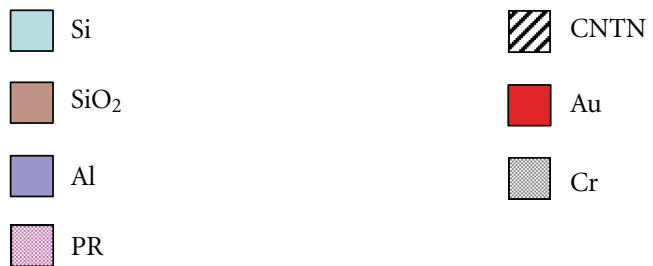

Figure 1: Schematic diagram of the fabrication process.

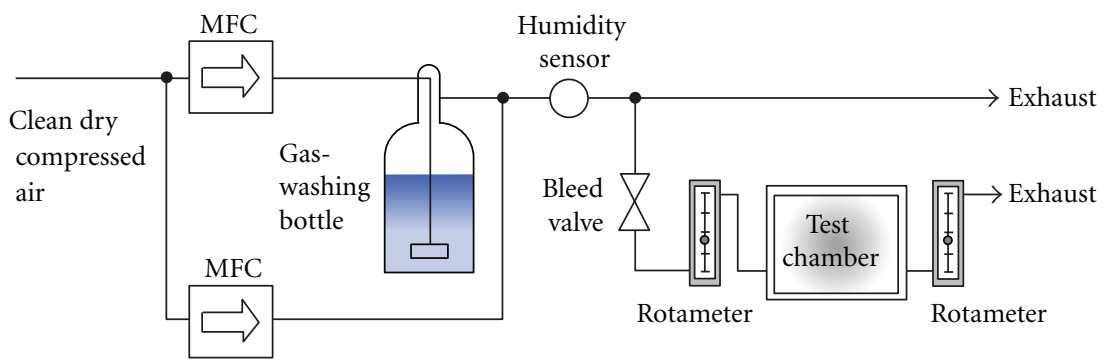

FIGURE 2: Schematic view of measurement setup (MFC-mass-flow controller, transistor chip is placed into the test chamber).

$I_{\mathrm{sd}}-V_{\mathrm{g}}$ (hereinafter $I-V$ ) dependence at different humidity conditions. As one can see after the humid air is introduced for $17 \mathrm{~min}$, the on-current (at negative gate voltage) is remarkably decreased. After switching the humidity OFF (closing the humid air valve) and keeping the chamber with the device in a dry air flow for $23 \mathrm{~min}$, the measured $I-V$ curve shows some recovery but does not completely return to its initial value. The possible explanation is that some of the water is not fully desorbed from the surface of the SWCNTs.

In order to investigate how fast the source-drain current $I_{\mathrm{sd}}$ responds to the humidity changes at different gate voltages, we kept dry air flow inside the chamber for $67 \mathrm{~min}$, after which we introduced the humid air into the chamber for the same period of time. Without applying the gate voltage
$\left(V_{g}=0 \mathrm{~V}\right)$, upon exposure to the humidity for $67 \mathrm{~min}$ a decrease of source-drain current, $I_{s d}$ was observed as shown in Figure 4(a). At negative gate voltages a larger current $I_{\mathrm{sd}}$ passes through the nanotube network. But at the same time, charge carriers are trapped in the dielectric layer of the FET. These charge traps are responsible for the hysteresis in the SWCNT transistor [27]. Considering the large area of trapping sites in the dielectric layer and the large number of SWCNTs in the random network transistors, the effect of charge trapping is more pronounced compared to an individual nanotube transistor. Positive charges are injected into the dielectric oxide traps at negative gate voltages and vice versa. The trapped charge is localized at the gate dielectric and SWCNT surface, and the charge is increased 


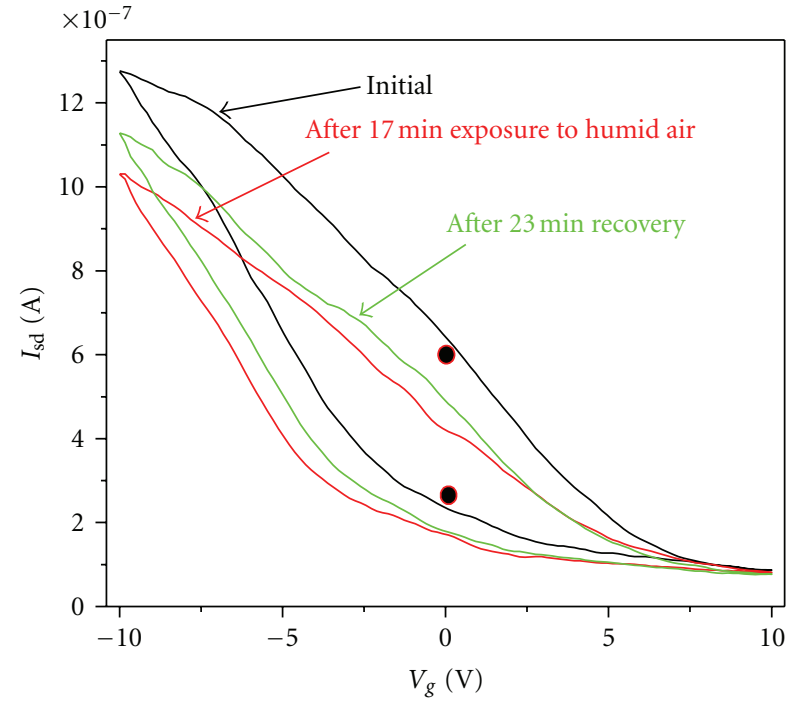

FIGURE 3: Source-drain current versus gate voltage measured in dry air (initial), after exposure in $78 \%$ humid air for $17 \mathrm{~min}$, and recovery after $23 \mathrm{~min}$ in dry air. The gate voltage is swept from $-10 \mathrm{~V}$ to $+10 \mathrm{~V}$ back to $-10 \mathrm{~V}$ at $V_{\mathrm{sd}}=600 \mathrm{mV}$.

with increasing the gate voltage. Due to the applied negative voltage, positive charge traps were formed. These charge traps act as a positive electric potential at SWCNT surface and reduced the effective negative gate voltage. This results in the increase of the depletion region, and hence to the $I_{\mathrm{sd}}$ decrease. At higher negative gate voltage $\left(V_{\mathrm{g}}=-10 \mathrm{~V}\right)$, more charge traps were formed compared to $V_{\mathrm{g}}=-6 \mathrm{~V}$, which led to larger decrease in the effective gate voltage, hence to a larger decrease in $I_{\mathrm{sd}}$. By exposing the FET device to humidity, free holes in the SWCNT network channel were neutralized (or compensated, $[21,22]$ ) with the electrons donated by the water molecules, and, thereby, a decrease in $I_{\text {sd }}$ current was observed at zero gate voltage. At higher negative gate voltages, this effect of charge carrier neutralization is stronger: therefore, the sharper decrease in the current was observed. $\mathrm{Na}$ et al. reported an inversion of the conductivity into $n$-type by increasing the relative humidity over $65 \%$ [22]. We have not observed this change, which can be likely explained by different nanotube type, density, and defectiveness. From the current dependence we can also evaluate the current decay time due to the humidity effect: about 30-50 min, decreasing at larger negative gate voltages.

At positive gate voltages, the current $I_{\mathrm{sd}}$ is low due to the increase of the depletion region, and the transistor is in OFF state (Figure 4(b)). At the same time, negative charge carriers can be trapped in the dielectric layer close to nanotubes. Keeping gate voltage constant in the initial dry air region results in the negative charge accumulation in the dielectric layer. The trapped negative charge can function as a negative potential reducing the effective positive gate bias. The reduction in the positive gate bias decreases the depletion region and as a result increases $I_{\text {sd }}$ (opposite to the effect described for the negative gate voltage). After switching the humidity on, we observed an increase in the

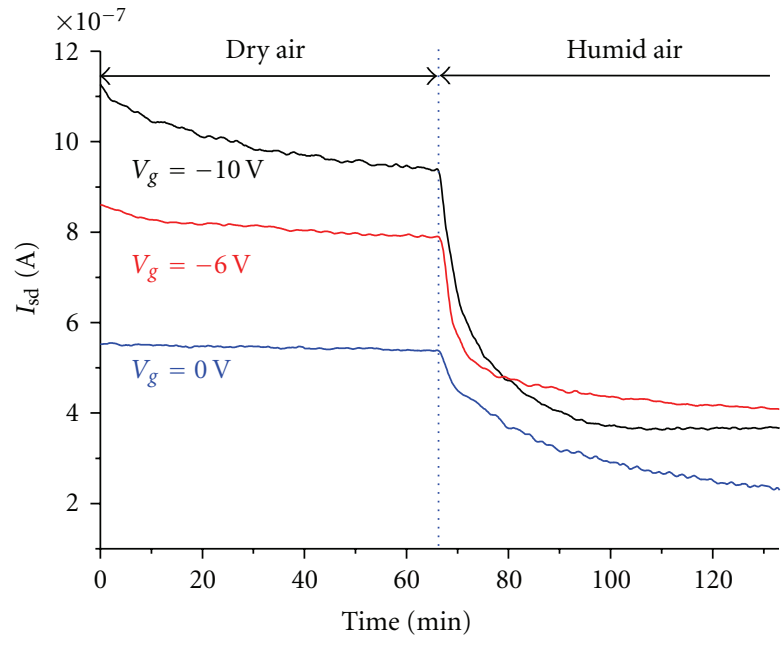

(a)

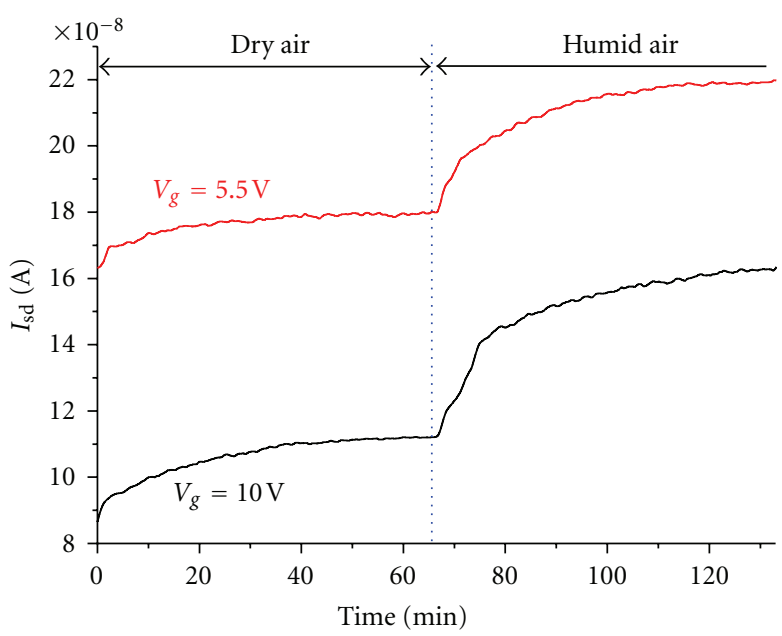

(b)

FIGURE 4: Source-drain current change of the FET sensor in dry and humid air $(\mathrm{RH}=78 \%)$ : at (a) negative and 0 gate voltages, and (b) positive gate voltages.

current $I_{\text {sd }}$. The rampup of the current is larger at higher gate voltages. The current increase can be explained by water molecule adsorption. Since at positive gate voltage the transistor channel is closed, the hole transport is suppressed. Therefore, electrons donated by water molecules increase both the number of charge carriers and current.

We have also studied the FET behaviour with a shorter exposure and recovery times. The humid air flow was introduced for $3 \mathrm{~min}$ followed by $4 \mathrm{~min}$ recovery time in dry air. The measurement results for negative and positive gate voltages are shown in Figure 5. At negative gate voltages we observed the largest absolute value of the current (since the transistor was in ON state), but the recovery was slower in this case (Figure 5(a)). In OFF state, that is, at positive gate voltage, the signal was lower, but the current pulses were sharper and almost symmetrical, with the recovery time of about 2 min (Figure 5(b)). At zero gate voltage we observed an intermediate shape of the current pulses due 
to the humidity change (Figure 5(a)) with practically no background current drift. The current drift during the measurements at shorter exposure times can be explained by the diffusion-limited penetration and adsorption/desorption of water molecules in the space between the SWCNTs, that is, inside bundles. Obviously, these structural changes affect the electrical contact between the tubes.

Comparing the above-mentioned current responses to the humidity pulses obtained at negative and positive gate voltages, let us discuss two different parameters of the sensors: sensitivity and speed. At negative gate voltage, the measured current pulses were about one order of magnitude higher compared to those measured at the positive gate voltage due to the fact that the transistor was in ON and OFF state, respectively. However, the relative levels of $I_{\text {sd }}$ changes were 8.2 and $8.7 \%$, respectively, confirming the identity of the observed phenomenon. At the same time, the recovery after closing the humid air was about three times faster when operating at positive gate voltage (less than $2 \mathrm{~min}$ ), compared to the negative gate (more than $3 \mathrm{~min}$ ). Most likely, this can be explained by larger charge trapped at negative gate voltage resulting in longer recovery. Thus, depending on the demand, different operation options for the humidity sensors based on SWCNTs can be proposed. If a larger signal is required, a FET humidity sensor based on the SWCNT networks should be operated at the negative gate voltages; if the sensing speed is important, then the FET should be utilized at the positive gate voltages.

It is worth noting that when the gate voltage is switched either from positive or negative gate to zero gate voltage, trapped charges still remained in the charge trapping sites resulting in the hysteresis of $I_{\mathrm{sd}}-V_{\mathrm{g}}$ curve (Figure 3). This charge trapping mechanism was effectively used in the fabrication of memory devices [28-31]. Therefore, at $V_{\mathrm{g}}=0$, transistor can be in ON or OFF state, depending on the direction of the gate voltage change. This kind of memory effect of previous gate voltages can affect the response to humidity, as shown in Figure 6. The device was kept in dry air at negative (Figure 6(a)) or positive (Figure 6(b)) gate voltages, and after switching to $V_{\mathrm{g}}=0$, either a current drop or increase happened, respectively (current values are corresponded to the solid dots shown in Figure 3). After the humid air was introduced, depending on the previous gate polarity, positive or negative current change was observed. Actually, the current changes to humidity seen in Figure 6 have the same polarity as current pulses presented in Figure 5. To conclude, the humidity FET device response at the gate voltages close to zero can be rather different, depending on the "history" of current-voltage sweeps. Therefore, it could be more reliable to operate the humidity sensors at higher gate voltages.

It is worth noting that compared to traditional sensors (e.g., based on metal oxides), the nanotube networks, especially random networks consisting of pristine highquality SWCNTs, have several features: inert outer walls, very small sizes (high specific surface area), and the presence of intertube contacts and intertube spaces, which apparently determine the conductivity and therefore the sensing properties of the transistors. In particular, the reversible adsorption/desorption of air components on/from the

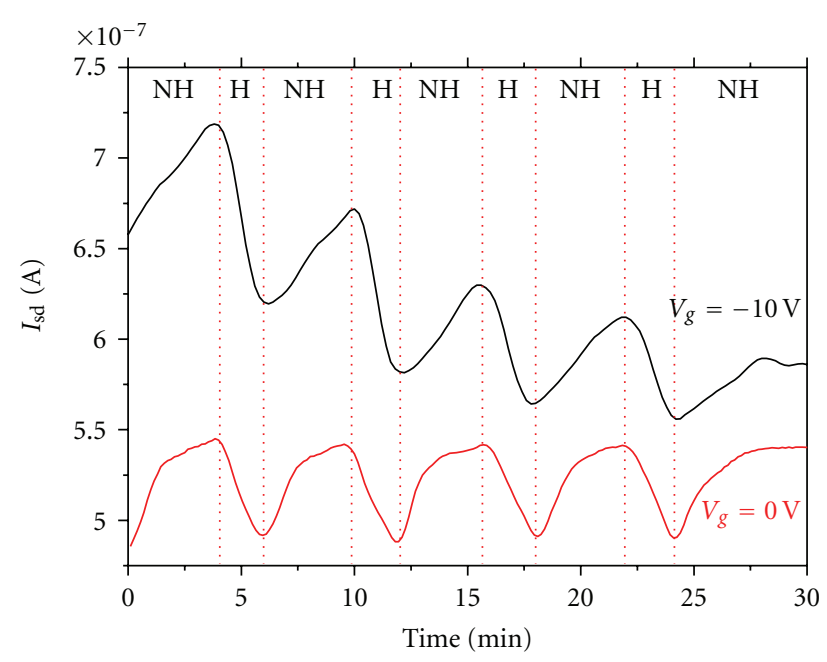

(a)

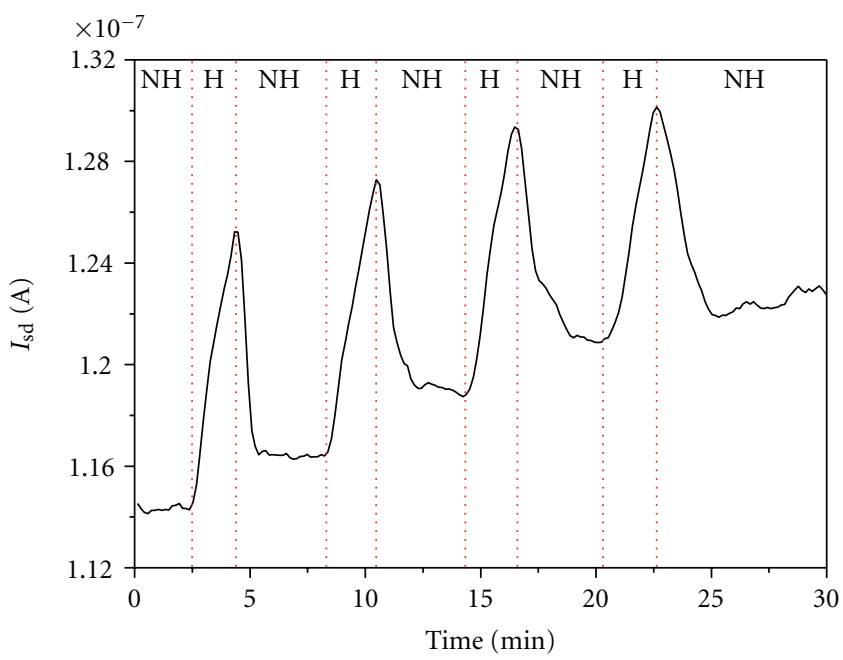

(b)

Figure 5: Current response to shorter humidity pulses monitored at: (a) $V_{\mathrm{g}}=0$ and $-10 \mathrm{~V}$, (b) $V_{\mathrm{g}}=10 \mathrm{~V}$. Sequence of $2 \mathrm{~min}$ humid air flow $(\mathrm{H})$ with 3 min recovery in dry air flow $(\mathrm{NH})$. $V_{\text {sd }}=600 \mathrm{mV}$.

surface and in/from the space between the tubes leads to longer stabilization time. For this reason, we believe that the SWCNT sensor performance can be significantly improved if aligned networks, preferably consisting of individual tubes, will be utilized between the electrodes.

\section{Conclusions}

We have fabricated SWCNT random network FETs and investigated the humidity effect on their source-drain current at different gate voltages. When exposed to humidity, the increase of the current at positive gate voltages and its decrease at negative gate voltages can be explained by the electron-donating nature of water molecules to SWCNTs. The open transistor circuit exposure to humidity at higher negative gate voltages suppressed the signal due to holes 


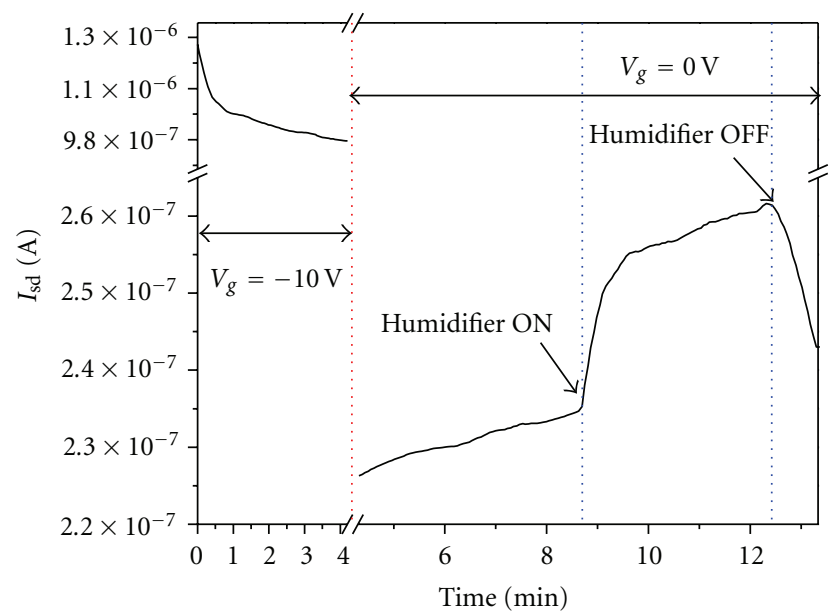

(a)

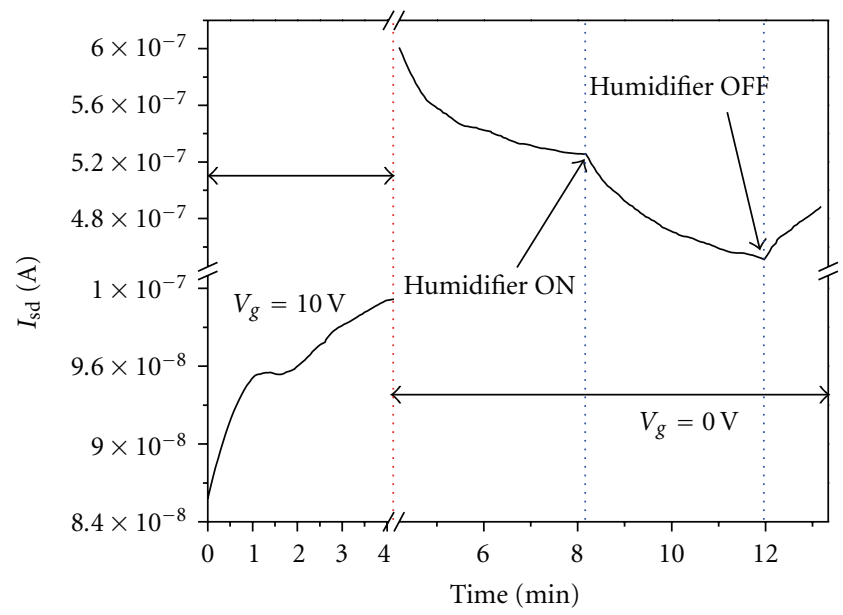

(b)

FIGURE 6: Monitoring current response to humidity at zero gate voltage: inversion of current change depending on previous gate voltage polarity (a) from $V_{\mathrm{g}}=-10$ to $0 \mathrm{~V}$, (b) from $V_{\mathrm{g}}=10$ to $0 \mathrm{~V}$.

compensated by electrons. At higher positive gate voltages upon exposure to humidity the transistors exhibited the current increase. Investigating the current response to short ( $2 \mathrm{~min}$ ) humid air pulses, we found that operation at negative gate voltages resulted in higher signals. Nevertheless, keeping the device at positive gate voltages allows about 2-3 times faster operation, but with a lower signal amplitude. The gate voltage can be adjusted in order to get the maximum sensing speed and recovery or amplitude and desired direction of the current pulses.

\section{Acknowledgments}

Markku Lahti from VTT Oulu and Sami Saukko from University of Oulu are gratefully acknowledged for fruitful discussions. The authors thank Marina Y. Timmermans for her assistance during the aerosol deposition of SWCNTs and for fruitful discussions. Financial support from Finnish National Graduate School in Nanoscience (NGS-NANO), Academy of Finland, and Nokia Nanosciences is acknowledged.

\section{References}

[1] S. Hong and S. Myung, "Nanotube electronics: a flexible approach to mobility," Nature Nanotechnology, vol. 2, no. 4, pp. 207-208, 2007.

[2] D. M. Guldi and N. Martín, Carbon Nanotubes and Related Structures: Synthesis, Characterization, Functionalization, and Applications, Wiley-VCH, Cambridge, UK, 2010.

[3] L. Hu, D. S. Hecht, and G. Gruner, "Percolation in transparent and conducting carbon nanotube networks," Nano Letters, vol. 4, no. 12, pp. 2513-2517, 2004.

[4] E. S. Snow, J. P. Novak, P. M. Campbell, and D. Park, "Random networks of carbon nanotubes as an electronic material," Applied Physics Letters, vol. 82, no. 13, pp. 2145-2147, 2003.

[5] M. C. LeMieux, M. Roberts, S. Barman, W. J. Yong, M. K. Jong, and Z. Bao, "Self-sorted, aligned nanotube networks for thinfilm transistors," Science, vol. 321, no. 5885, pp. 101-104, 2008.

[6] A. G. Nasibulin, A. Ollikainen, A. S. Anisimov et al., "Integration of single-walled carbon nanotubes into polymer films by thermo-compression," Chemical Engineering Journal, vol. 136, no. 2-3, pp. 409-413, 2008.

[7] A. Kaskela, A. G. Nasibulin, M. Y. Timmermans et al., "Aerosol-synthesized SWCNT networks with tunable conductivity and transparency by a dry transfer technique," Nano Letters, vol. 10, no. 11, pp. 4349-4355, 2010.

[8] D.-M. Sun, M. Y. Timmermans, Y. Tian et al., "Flexible highperformance carbon nanotube integrated circuits," Nature Nanotechnology, vol. 6, no. 3, pp. 156-161, 2011.

[9] K. Bradley, J. C. P. Gabriel, and G. Grüner, "Flexible nanotube electronics," Nano Letters, vol. 3, no. 10, pp. 1353-1355, 2003.

[10] M. W. Rowell, M. A. Topinka, M. D. McGehee et al., "Organic solar cells with carbon nanotube network electrodes," Applied Physics Letters, vol. 88, no. 23, Article ID 233506, 2006.

[11] C. Wang, J. Zhang, K. Ryu, A. Badmaev, L. G. De Arco, and C. Zhou, "Wafer-scale fabrication of separated carbon nanotube thin-film transistors for display applications," Nano Letters, vol. 9, no. 12, pp. 4285-4291, 2009.

[12] Z. Li, H. R. Kandel, E. Dervishi et al., "Comparative study on different carbon nanotube materials in terms of transparent conductive coatings," Langmuir, vol. 24, no. 6, pp. 2655-2662, 2008.

[13] A. Kaskela, A. G. Nasibulin, M. Y. Timmermans et al., "Aerosol-synthesized SWCNT networks with tunable conductivity and transparency by a dry transfer technique," Nano Letters, vol. 10, no. 11, pp. 4349-4355, 2010.

[14] J. T. W. Yeow and Y. Wang, "A review of carbon nanotubesbased gas sensors," Journal of Sensors, vol. 2009, Article ID 493904, 24 pages, 2009.

[15] C. L. Cao, C. G. Hu, L. Fang, S. X. Wang, Y. S. Tian, and C. Y. Pan, "Humidity sensor based on multi-walled carbon nanotube thin films," Journal of Nanomaterials, vol. 2011, Article ID 707303, 2011.

[16] W. F. Jiang, S. H. Xiao, C. Y. Feng, H. Y. Li, and X. J. Li, "Resistive humidity sensitivity of arrayed multi-wall carbon nanotube nests grown on arrayed nanoporous silicon pillars," Sensors and Actuators B, vol. 125, no. 2, pp. 651-655, 2007.

[17] Z. Zhao, X. Liu, X. Wang, T. Li, and W. Wang, "Stability of humidity sensor based on carbon nanotubes at different frequencies," in 2010 Academic Symposium on Optoelectronics and Microelectronics Technology and 10th Chinese-Russian Symposium on Laser Physics and Laser Technology, RCSLPLT/ASOT 2010, pp. 322-325, chn, August 2010.

[18] W. Jiang, S. Xiao, H. Zhang, Y. Dong, and X. Li, "Capacitive humidity sensing properties of carbon nanotubes grown on 
silicon nanoporous pillar array," Science in China E, vol. 50, no. 4, pp. 510-515, 2007.

[19] A. Star, T. R. Han, V. Joshi, and J. R. Stetter, "Sensing with nafion coated carbon nanotube field-effect transistors," Electroanalysis, vol. 16, no. 1-2, pp. 108-112, 2004.

[20] L. Liu, X. Ye, K. Wu, R. Han, Z. Zhou, and T. Cui, "Humidity sensitivity of multi-walled carbon nanotube networks deposited by dielectrophoresis," Sensors, vol. 9, no. 3, pp. 1714-1721, 2009.

[21] A. Zahab, L. Spina, P. Poncharal, and C. Marlière, "Watervapor effect on the electrical conductivity of a single-walled carbon nanotube mat," Physical Review B, vol. 62, no. 15, pp. 10000-10003, 2000.

[22] P. S. Na, H. Kim, H. M. So et al., "Investigation of the humidity effect on the electrical properties of single-walled carbon nanotube transistors," Applied Physics Letters, vol. 87, no. 9, pp. $1-3,2005$.

[23] Y. Tian, M. Y. Timmermans, M. Partanen et al., "Growth of single-walled carbon nanotubes with controlled diameters and lengths by an aerosol method," Carbon, 2011.

[24] M. Y. Timmermans, K. Grigoras, A. G. Nasibulin et al., "Lithography-free fabrication of carbon nanotube network transistors," Nanotechnology, vol. 22, no. 6, Article ID 065303, 2011.

[25] M. Y. Zavodchikova, T. Kulmala, A. G. Nasibulin et al., "Carbon nanotube thin film transistors based on aerosol methods," Nanotechnology, vol. 20, no. 8, Article ID 085201, 2009.

[26] R. Martel, T. Schmidt, H. R. Shea, T. Hertel, and P. Avouris, "Single- and multi-wall carbon nanotube field-effect transistors," Applied Physics Letters, vol. 73, no. 17, pp. 2447-2449, 1998.

[27] M. Rinkiö, M. Y. Zavodchikova, P. Törmä, and A. Johansson, "Effect of humidity on the hysteresis of single walled carbon nanotube field-effect transistors," Physica Status Solidi B, vol. 245, no. 10, pp. 2315-2318, 2008.

[28] M. Radosavljević, M. Freitag, K. V. Thadani, and A. T. Johnson, "Nonvolatile molecular memory elements based on ambipolar nanotube field effect transistors," Nano Letters, vol. 2, no. 7, pp. 761-764, 2002.

[29] W. Kim, A. Javey, O. Vermesh, Q. Wang, Y. Li, and H. Dai, "Hysteresis caused by water molecules in carbon nanotube field-effect transistors," Nano Letters, vol. 3, no. 2, pp. 193-198, 2003.

[30] W. Sigen, S. Paul, Z. Qing, and Y. Dajiang, "Nonvolatile memory from single-walled carbon nanotube-based field effect transistors," Current Nanoscience, vol. 1, no. 1, pp. 43-46, 2005.

[31] M. Rinkiö, A. Johansson, M. Y. Zavodchikova et al., "Highyield of memory elements from carbon nanotube field-effect transistors with atomic layer deposited gate dielectric," New Journal of Physics, vol. 10, Article ID 103019, 2008. 

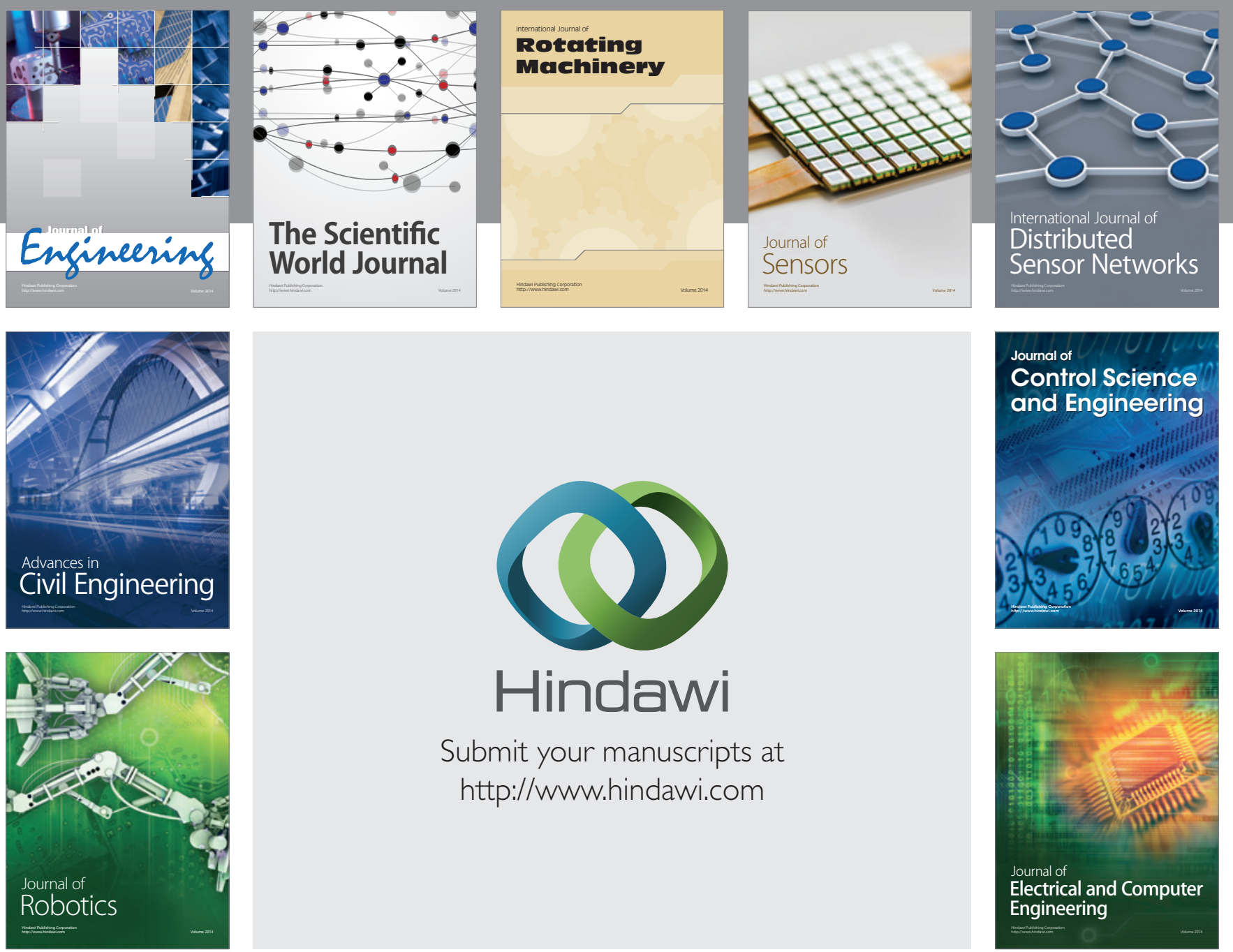

Submit your manuscripts at

http://www.hindawi.com
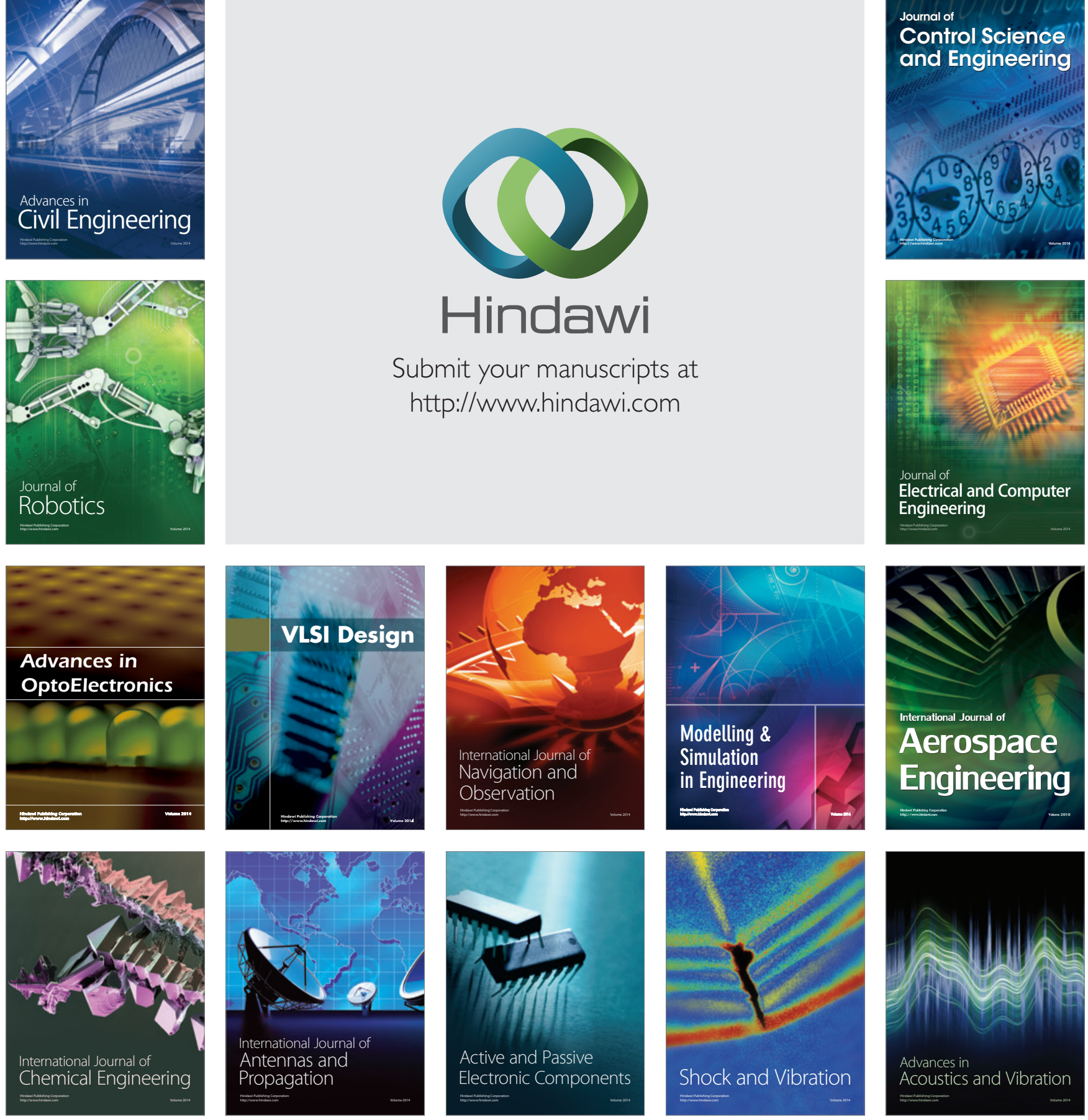